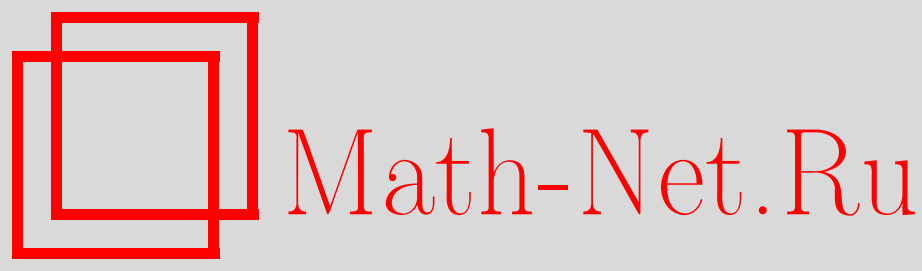

М. В. Комарова, М. Ю. Налимов, Ю. Хонконен, Температурные функции Грина в ферми-системах: сверхпроводящий фазовый переход, ТМФ, 2013, том 176, номер 1, 89-97

DOI: https://doi.org/10.4213/tmf8486

Использование Общероссийского математического портала Math-Net.Ru подразумевает, что вы прочитали и согласны с пользовательским соглашением http://www . mathnet.ru/rus/agreement

Параметры загрузки:

IP : 54.209 .52 .79

26 апреля 2023 г., $11: 17: 13$

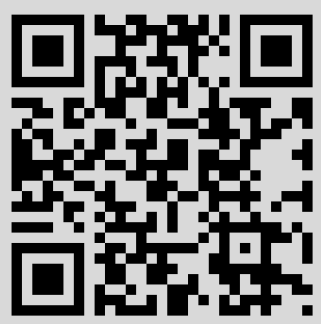




\title{
ФИЗИКА
}

Том 176, № 1

июль, 2013

(C) 2013 г. М. М. Комарова*, М. Ю. Налимов*, Ю. Хонконен ${ }^{\dagger}$

\section{ТЕМПЕРАТУРНЫЕ ФУНКЦИИ ГРИНА В ФЕРМИ-СИСТЕМАХ: СВЕРХПРОВОДЯЩИЙ ФАЗОВЫЙ ПЕРЕХОД}

\begin{abstract}
Рассматривается модель равновесной ферми-системы частиц с произвольным спином и взаимодействием типа плотность-плотность. На основе микроскопического гамильтониана в формализме температурных функций Грина определены критические моды и построено эффективное действие, описывающее окрестность точки фазового перехода. Ренормгрупповой анализ полученной модели приводит к стандартным показателям критического поведения для случая фермионов со спином $1 / 2$, однако он показывает, что в системе фермионов с большим спином имеет место фазовый переход первого рода, причем его температура превышает стандартные оценки для температуры фазового перехода второго рода.
\end{abstract}

Ключевые слова: температурные функции Грина, сверхпроводимость, критическое поведение, ренормализационная группа.

DOI: $10.4213 / \operatorname{tmf} 8486$

\section{1. ВВЕДЕНИЕ}

Исследованию квантовых систем ферми-частиц уделяется постоянное внимание. Особый интерес вызывает наличие в данных системах фазового перехода в сверхпроводящее состояние. Описанию этого фазового перехода и посвящена настоящая работа.

Существуют два принципиально разных подхода к рассматриваемой проблеме. Первый основан на микроскопическом описании квантового газа с взаимодействием типа плотность-плотность [1], он позволяет в рамках теории возмущений обнаружить явление сверхпроводимости и вычислить температуру фазового перехода. Одной из проблем данного описания является отсутствие в нем теории среднего поля как нулевого приближения для свободной энергии, поскольку такая теория не может быть построена в грассмановых переменных.

Второй подход, касающийся описания малой окрестности перехода, аппелирует, по сути, к гамильтониану Гинзбурга-Ландау, т. е. к эффективной теории типа $\varphi^{4}$,

* Санкт-Петербургский государственный университет, Санкт-Петербург, Россия. E-mail: frommarina@mail.ru, mynalimov@gmail.com

${ }^{\dagger}$ National Defence University, Helsinki, Finland. E-mail: juha.honkonen@helsinki.fi 
построенной для предполагаемого параметра порядка [2], [3]. Теория среднего поля в таком подходе также написана исходя из общих соображений о структуре параметра порядка. Модель исследуется в окрестности точки перехода методом ренормализационной группы (РГ), и для системы электронов со взаимодействием данный анализ приводит к критическим индексам, соответствующим $\varphi^{4}(n=2)$ модели.

В настоящей работе мы подробно прослеживаем связь между этими двумя подходами. В разделе 2 используется формализм температурных функций Грина для описания равновесной квантовой ферми-системы. Начиная с простейшей микроскопической модели с бесструктурным взаимодействием типа плотность-плотность, мы вводим новые поля, которые оказываются критическими модами модели, а параметры порядка - их средними значениями.

В разделе 3 мы строим инфракрасно-эффективное (ИК-эффективное) действие, описывающее поведение в окрестности точки перехода, и показываем, что теория среднего поля, демонстрирующая существование и характер фазового перехода, легко может быть построена во введенных переменных - критических модах. Построенное эффективное действие исследуется в рамках РГ-подхода на основе $(4-\epsilon)$-разложения для описания окрестности точки фазового перехода. В результате проведенного анализа установлена существенная зависимость характера фазового перехода от спина фермионов: для частиц со спином $1 / 2$ в системе наблюдается фазовый переход второго рода с критическими индексами, соответствующими $\varphi^{4}(n=2)$ теории (как и ожидалось), однако фермионы с высшим спином демонстрируют фазовый переход первого рода, имеющий место при более высокой температуре, но приводящий также в сверхпроводящее состояние.

\section{2. КРИТИЧЕСКИЕ МОДЫ СВЕРХПРОВОДЯЩЕГО ФАЗОВОГО ПЕРЕХОДА}

Наш анализ основан на квантово-полевой модели с действием

$$
S=\psi_{i}^{+}\left(\partial_{t}-\frac{\Delta}{2 m}-\mu\right) \psi_{i}-\frac{\lambda}{2}\left(\psi_{i}^{+} \psi_{i}\right)\left(\psi_{j}^{+} \psi_{j}\right)
$$

Здесь $\psi_{i}(\mathbf{x}, t), \psi_{i}^{+}(\mathbf{x}, t)$ - комплексно-сопряженные грассмановы переменные, связанные по своей природе с фермионными волновыми функциями, $i=1, \ldots, r$ - число возможных значений проекции спина, $\mathbf{x}$ - координата, $t$ - "мнимое время" (евклидов разворот), изменяющееся в интервале $[0, \beta=1 /(k T)]$, зависящем от температуры, $\mu$ - химический потенциал, $\lambda$ - константа взаимодействия. На поля $\psi, \psi^{+}$наложены антипериодические граничные условия по $t$. Все необходимые интегрирования по $t$ и $\mathbf{x}$ в формуле (1) и аналогичных формулах в дальнейшем подразумеваются. В формуле (1) используется приближение (по сути не являющееся существенным для приведенного анализа) локального взаимодействия типа плотность-плотность, т. е. стандартное приближение для описания критического поведения [1], [4].

В модели (1) температура фазового перехода определяется появлением аномального решения уравнения Дайсона

$$
G^{-1}=G_{0}^{-1}-\Sigma,
$$


где $G$ - полная двухвостая функция Грина, $G_{0}$ - свободная функция Грина, а $\Sigma-$ сумма всех собственно-энергетических графов [1]. Отметим, что пропагаторы полей $\psi^{+}, \psi$ при этой температуре остаются массивными, поэтому эти поля не являются критическими модами теории в том смысле, что они не определяют критические сингулярности. Не определяют они также и параметры порядка, так как теория среднего поля не может быть построена в модели (1), средние значения грассмановых полей $\psi^{+}, \psi$ всегда равны нулю.

Как известно, параметром порядка сверхпроводящего фазового перехода являются средние значения составных операторов $\langle\psi \psi\rangle,\left\langle\psi^{+} \psi^{+}\right\rangle$; скобки $\langle\cdot\rangle$ означают усреднение с весом $e^{-S}$. Чтобы описать фазовый переход в модели (1), введем вспомогательные поля $\chi_{i j}, \chi_{i j}^{+}$(преобразование Хаббарда-Стратоновича в стиле [2]) и перепишем действие (1) в виде

$$
S=\psi_{i}^{+}\left(\partial_{t}-\frac{\Delta}{2 m}-\mu\right) \psi_{i}+\chi_{j i}^{+} \chi_{i j}+\sqrt{\frac{\lambda}{2}} \chi_{i j}\left(\psi_{i}^{+} \psi_{j}^{+}\right)+\sqrt{\frac{\lambda}{2}} \chi_{i j}^{+}\left(\psi_{i} \psi_{j}\right) .
$$

Гауссово интегрирование $e^{-S}$ с действием (3) по полям $\chi_{i j}, \chi_{i j}^{+}$возвращает нас к действию (1). Поля $\psi, \psi^{+}$грассмановы, поэтому поля $\chi, \chi^{+}$можно рассматривать как комплексные антисимметричные матрицы ранга $r$.

Рассмотрим уравнения Швингера [4] в теории (3):

$$
\begin{aligned}
\left\langle\left(\partial_{t}-\frac{\Delta}{2 m}-\mu\right) \psi_{i}+\sqrt{2 \lambda} \chi_{i j} \psi_{j}^{+}\right\rangle & =A_{\psi i} \\
\left\langle\left(-\partial_{t}-\frac{\Delta}{2 m}-\mu\right) \psi_{i}^{+}+\sqrt{2 \lambda} \chi_{i j}^{+} \psi_{j}\right\rangle & =A_{\psi+i} \\
\left\langle\chi_{i j}^{+}-\sqrt{\frac{\lambda}{2}} \psi_{i}^{+} \psi_{j}^{+}\right\rangle & =A_{\chi+i j}, \\
\left\langle\chi_{j i}+\sqrt{\frac{\lambda}{2}} \psi_{i} \psi_{j}\right\rangle & =A_{\chi i j}
\end{aligned}
$$

где $A_{\phi}$ обозначает источники соответствующих полей, $\phi=\left\{\psi, \psi^{+}, \chi, \chi^{+}\right\}$. Последние два уравнения (при физических значениях $A_{\phi}=0$ ) показывают, что средние значения полей $\chi^{+}, \chi$ непосредственно связаны с параметром порядка.

Введем обозначения $\alpha_{i} \equiv\left\langle\psi_{i}\right\rangle, \beta_{i j} \equiv\left\langle\chi_{i j}\right\rangle, \alpha_{i}^{+} \equiv\left\langle\psi_{i}^{+}\right\rangle, \beta_{i j}^{+} \equiv\left\langle\chi_{i j}^{+}\right\rangle ; \Gamma-$ производящий функционал 1-неприводимых функций Грина (функционал первого преобразования Лежандра) [4]. Покажем, что поля $\chi^{+}, \chi$ и являются критическими модами в указанном выше смысле. Для этого рассмотрим следующие из уравнений (4) уравнения стационарности Лежандра [4]

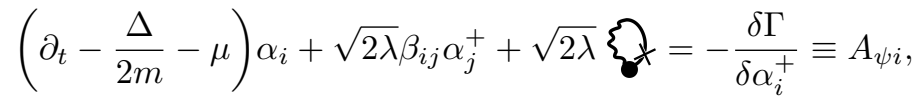

$$
\begin{aligned}
& \left(-\partial_{t}-\frac{\Delta}{2 m}-\mu\right) \alpha_{i}^{+}+\sqrt{2 \lambda} \beta_{i j}^{+} \alpha_{j}+\sqrt{2 \lambda} \underbrace{}_{0}=-\frac{\delta \Gamma}{\delta \alpha_{i}} \equiv A_{\psi+i}, \\
& \beta_{i j}^{+}-\sqrt{\frac{\lambda}{2}} \alpha_{i}^{+} \alpha_{j}^{+}-\sqrt{\frac{\lambda}{2}} \widehat{\chi x}=-\frac{\delta \Gamma}{\delta \beta_{j i}} \equiv A_{\chi+i j}, \\
& \beta_{j i}+\sqrt{\frac{\lambda}{2}} \alpha_{i} \alpha_{j}+\sqrt{\frac{\lambda}{2}} \bigcirc=-\frac{\delta \Gamma}{\delta \beta_{j i}^{+}} \equiv A_{\chi i j} .
\end{aligned}
$$


Полям $\psi, \psi^{+}$здесь соответствуют обычные линии, $\chi, \chi^{+}-$волнистые, крестик на линии соответствует полю с крестом, все линии в графах здесь и в дальнейшем в данном разделе подразумеваются одетыми, индексы линий в графах опущены, но могут быть легко восстановлены в соответствии с уравнениями (4).

Решением этих уравнений при $A_{\phi}=0$ являются $\alpha_{i}=\alpha_{i}^{+}=0$ (всегда); $\beta=\beta^{+}=$ 0 в неупорядоченной фазе и $\beta \neq 0, \beta^{+} \neq 0$ (т.е. образование конденсата) после фазового перехода.

Варьируя уравнения (5) по полям, несложно получить матрицу обратных пропагаторов $-\delta^{2} \Gamma / \gamma, \gamma=\{\alpha, \beta\}$, а по ней определить сами пропагаторы полей. Следует отметить, что пропагаторы $\left\langle\chi^{+} \psi\right\rangle,\left\langle\chi \psi^{+}\right\rangle,\left\langle\psi^{+} \psi^{+}\right\rangle,\langle\psi \psi\rangle$ при физических значениях $\alpha$ и $\beta$ в неупорядоченной фазе равны нулю. В упорядоченной фазе эти пропагаторы стремятся к нулю в окрестности точки перехода, когда $\beta \rightarrow 0$ и $\beta^{+} \rightarrow 0$, и могут быть разложены в ряды по $\beta, \beta^{+}$. Поэтому, разложив в ряд по $\beta_{l k}$, например, последнее уравнение из системы (5) при $A_{\phi}=0$, можно утверждать, что критической точке соответствует появление нетривиального решения уравнения

$$
\beta_{j i}+\sqrt{\frac{\lambda}{2}} \bigodot_{\gamma}^{\xi} \beta+O\left(\beta^{2}\right)=0
$$

(при получении данного уравнения использовано соотношение

$$
\partial_{\beta} \quad=-\frac{\xi}{-}
$$

в котором вершина полагается одетой).

Теперь функция Грина $\left\langle\chi^{+} \chi\right\rangle$ в окрестности точки фазового перехода определяется соотношением (5):

$$
\left(-\frac{\delta^{2} \Gamma}{\delta \beta \delta \beta^{+}}\right)^{-1}=\left\langle\chi^{+} \chi\right\rangle^{-1}=1+\sqrt{\frac{\lambda}{2}}
$$

(матрица обратных пропагаторов обращается в данном приближении тривиально) и в соответствии с решением (6) действительно оказывается безмассовой в критической точке.

Тем самым установлено, что поля $\chi$ и $\chi^{+}-$безмассовые критические моды в точке фазового перехода. Используя первое преобразование Лежандра, в этих переменных можно описывать фазовый переход так же, как и в бозонном случае; можно построить теорию среднего поля и петлевые поправки к ней.

\section{3. ЭФФЕКТИВНОЕ ДЕЙСТВИЕ И КРИТИЧЕСКОЕ ПОВЕДЕНИЕ}

Чтобы получить эффективное действие, описывающее поведение модели в окрестности точки перехода, введем в теории (3) члены с источниками полей $A_{\psi}, A_{\psi+}$ и выполним гауссово интегрирование по полям $\psi, \psi^{+}$. В результате получаем теорию с действием

$$
S=\chi_{j i}^{+} \chi_{i j}+\frac{1}{2} m \bigcirc m+\frac{1}{3} \int_{m}^{\xi}+\frac{1}{4} m_{m}^{m}+\cdots+A_{\psi} G A_{\psi+},
$$


где волнистыми хвостиками обозначены поля $\chi, \chi^{+}$. В петлевых вкладах в действие (8) подразумевается операция взятия следа по индексам,

$$
G=\quad+\underline{\xi}+\underline{\xi}+\cdots
$$

(в выражении (8) и в последнем равенстве линиями обозначены затравочные пропагаторы). Заметим, что $\operatorname{Tr}\left(\chi \chi^{+} \chi\right)=0$, соответствующие диаграммы просто не существуют. Функционал $G$ позволяет в теории (8) строить корреляционные функции исходных полей $\psi^{+}, \psi$ и показывает, что критические поправки в функциях Грина этих полей определяются разнообразными составными операторами полей $\chi$, $\chi^{+}$. Предполагая $\Lambda$-регуляризацию в графах действия (8), эффективное действие следует строить в форме гамильтониана Гинзбурга-Ландау, вычисляя все графики в виде разложения по внешним импульсам и частотам. Поля $\chi, \chi^{+}$бозонные с периодическими граничными условиями $\left.\chi^{(+)}(t)\right|_{t=0}=\left.\chi^{(+)}(t)\right|_{t=\beta}$. Рассмотрев пропагаторы этих полей и отобрав лишь ИК-существенные члены в них, можно убедиться, что в критической области эти поля следует считать $t$-независимыми. Тогда после некого конечного растяжения полей эффективное действие приобретает вид

$$
S=\chi_{j i}^{+}(-\Delta) \chi_{i j}+\tau \chi_{j i}^{+} \chi_{i j}+\frac{g_{1}}{2}\left(\operatorname{Tr}\left(\chi^{+} \chi\right)\right)^{2}+\frac{g_{2}}{2} \operatorname{Tr}\left(\chi^{+} \chi \chi^{+} \chi\right)+A_{\psi} G A_{\psi+} .
$$

Напомним, что поля $\chi$ - антисимметричные матрицы ранга $r$. Заметим, что для мультипликативной ренормируемости теории в действие приходится включить взаимодействие с зарядом $g_{1}$. Несложно убедиться, что соответствующие контрчлены возникают в процессе ренормировки теории, начиная с простейшей однопетлевой диаграммы, что, впрочем, соответствует трехпетлевому вкладу в терминах исходных полей $\psi, \psi^{+}$.

При рассмотрении системы обычных электронов $r=2$ (при этом члены взаимодействия в формуле (9) оказываются линейно зависимыми) можно ограничиться рассмотрением формулы (9) с $g_{2}=0$. Таким образом, мы получаем обычную $\varphi^{4}$-модель со стандартными критическими индексами, соответствующими $n=$ 2. Здесь уместно напомнить, что антисимметричные матрицы ранга 2 однозначно задаются единственным комплексным числом.

Для фермионов со старшими спинами $r=4,6, \ldots$ члены действия (9) с коэффициентами $g_{1}, g_{2}$ линейно независимы, действие содержит обе независимые константы связи. Учитывая, что поля $\chi^{+}$и $\chi-$ комплексно-сопряженные матрицы, несложно получить условие устойчивости (положительной определенности) действия в виде ${ }^{1)}$

$$
g_{2}+r g_{1}>0
$$

при $g_{2}>0$. Отметим, что вследствие (8) затравочные значения зарядов $g_{20}>0$, $g_{10}=0$. Обычно в фермионных системах параметр Гинзбурга-Леванюка мал,

1) Для получения данного неравенства мы можем рассмотреть очевидное неравенство $\operatorname{Tr}\left(\chi^{+} \chi+\right.$ $\left.e\left(\operatorname{Tr} \chi^{+} \chi\right) I\right)^{2} \geqslant 0$, где $I-$ единичная матрица, $e=-1 / r$. Равенство реализуется на блочно-антисимметричных матрицах с коэффициентами \pm 1 . При $g_{2}<0$ область положительной определенности действия можно определить, приведя матрицы $\chi^{+}, \chi$ к той же пфаффовой форме. В результате имеем $g_{2}+r g_{1}>0$. 
поэтому имеются основания полагать, что физическим значением следует признать $g_{2}>0$.

Теория (9) ренормируется мультипликативно. Используя размерную регуляризацию в пространстве $4-\epsilon$, сосчитаем константы ренормировки зарядов и напишем уравнения РГ. В однопетлевом приближении бета-функции теории имеют вид

$$
\begin{aligned}
& \beta^{(g 1)}=-\epsilon g_{1}+\frac{1}{2}\left(r^{2}-r+8\right) g_{1}^{2}+2(r-1) g_{1} g_{2}+\frac{3}{2} g_{2}^{2}, \\
& \beta^{(g 2)}=-\epsilon g_{2}+6 g_{1} g_{2}+\frac{1}{2}(2 r-5) g_{2}^{2}
\end{aligned}
$$

(использовано растяжение зарядов $g_{i} \rightarrow g_{i} / 16 \pi^{2}$ ).

Кроме тривиальной фиксированной точки $g_{1 *}=g_{2 *}=0$ (ИК-неустойчивой при положительных $\epsilon$ ) в этой системе имеется нетривиальная фиксированная точка $g_{2 *}=0, g_{1 *}=2 \epsilon /\left(r^{2}-r+8\right)$. Индексы $\omega$ в этой точке суть $\epsilon$ и $\left(12 /\left(r^{2}-r+8\right)-1\right) \epsilon$. Поэтому она оказывается ИК-устойчивой в случае $r=2$, соответствующем обычным фермионам со спином $1 / 2$. В случае $r>2$ эта точка ИК-неустойчива.

Еще две нетривиальные фиксированные точки имеют координаты

$$
\begin{aligned}
& g_{1 *}=\frac{-4 r^{2}+8 r+77 \pm \sqrt{-32 r^{4}+224 r^{3}-324 r^{2}-580 r+1225}}{4 r^{4}-24 r^{3}-19 r^{2}+151 r+392} \epsilon, \\
& g_{2 *}=\frac{2\left(\epsilon-6 g_{1 *}\right)}{2 r-5}
\end{aligned}
$$

Они вещественны лишь при $r=2$ (при четных $r$ ), но либо ИК-неустойчивы, либо лежат в нефизической области $g_{2}<0$.

Таким образом, в случае $r>2$ ситуация нетривиальна. Здесь система (11) вовсе не имеет ИК-устойчивых фиксированных точек. Обычно это означает, что, строго говоря, РГ-подход неприменим. Часто в таких случаях высказывается предположение, что в системе происходит фазовый переход первого рода. Но в рассматриваемом случае нам удалось сделать более строгое утверждение.

Изучим поведение системы при стремлении температуры к $T_{\mathrm{c}}$. Вследствие уравнения (6) (или эквивалентного ему (2)) переменная $\tau \sim T-T_{\text {с }}$ (9) пропорциональна отличию правой части уравнения (7) от нуля при нулевых внешних импульсе и частоте. Безразмерной переменной, определяющей отклонение системы от критической точки, теперь будет $s=\tau / M, M$ - ренормировочная масса. Введем динамическую переменную уравнения РГ как $\zeta=\ln s$ и рассмотрим уравнения для инвариантных зарядов $\bar{g}_{1}, \bar{g}_{2}: \partial_{\zeta} \bar{g}_{i}=\beta^{\left(g_{i}\right)}\left(\bar{g}_{1}, \bar{g}_{2}\right)$.

Чтобы получить возможность использовать результаты однопетлевых расчетов, проведем растяжение зарядов и динамической переменной уравнения РГ: $\bar{g}_{i}=\epsilon \bar{g}_{i}^{\prime}$, $\zeta^{\prime}=\zeta / \epsilon$, параметр $\epsilon$ полагаем формально малым. Теперь параметр $\epsilon$ исключается из уравнений РГ для инвариантных зарядов в однопетлевом приближении, он возникает в разных степенях как множители при вкладах в бета-функции от диаграмм 


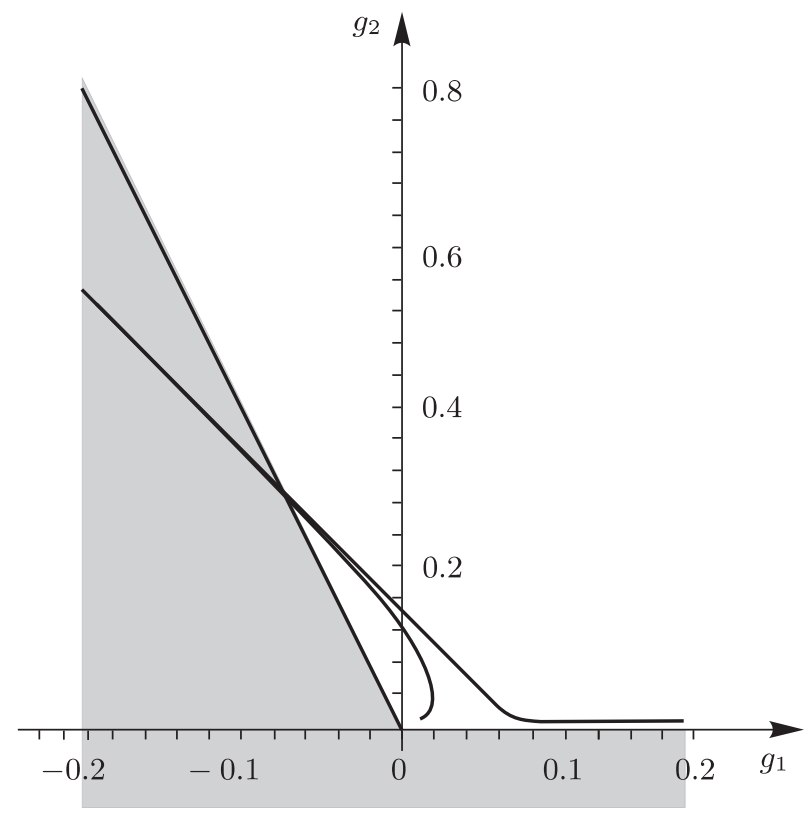

Рис. 1. Траектории инвариантных зарядов, стартующих из разных начальных точек в области $g_{1}>0, g_{2}>0$. В заштрихованной области утрачивается положительная определенность действия.

старших порядков. Уравнения для инвариантных зарядов приобретают вид

$$
\begin{aligned}
& \partial_{\zeta} \bar{g}_{1}^{\prime}=-\bar{g}_{1}^{\prime}+\frac{1}{2}\left(r^{2}-r+8\right) \bar{g}_{1}^{, 2}+2(r-1) \bar{g}_{1}^{\prime} \bar{g}_{2}^{\prime}+\frac{3}{2} \bar{g}_{2}^{, 2}+O(\epsilon), \\
& \partial_{\zeta} \bar{g}_{2}=-\bar{g}_{2}^{\prime}+6 \bar{g}_{1}^{\prime} \bar{g}_{2}^{\prime}+\frac{1}{2}(2 r-5) \bar{g}_{2}^{, 2}+O(\epsilon),
\end{aligned}
$$

и мы получаем возможность строить $\epsilon$-разложение в системе без устойчивой фиксированной точки. Результаты численного анализа системы уравнений в ведущем порядке по $\epsilon$ для случая $r=4$ приведены на рис. 1.

Отметим, что инвариантные заряды, стартуя с разных начальных значений, пересекают примерно по одной траектории границу области устойчивости действия (10), т. е. несмотря на отсутствие устойчивой фиксированной точки в системе реализуется примерная универсальность.

Таким образом, в рамках $\epsilon$-разложения нами получено строгое указание на существование в системе фазового перехода первого рода. Действительно, рассмотрим уравнение теории среднего поля для полей $\chi^{+}, \chi$ :

$$
(\Delta+\tau) \beta+g_{1} \operatorname{Tr}\left(\beta^{+} \beta\right) \beta+g_{2} \beta \beta^{+} \beta=0 .
$$

Как известно, оно является древесным приближением для более точного уравнения стационарности первого преобразования Лежандра в модели с действием (9). Различные петлевые вклады в этом уравнении содержат ИК-сингулярности, собираемые методом РГ в инвариантные переменные. После такой обработки вклады 


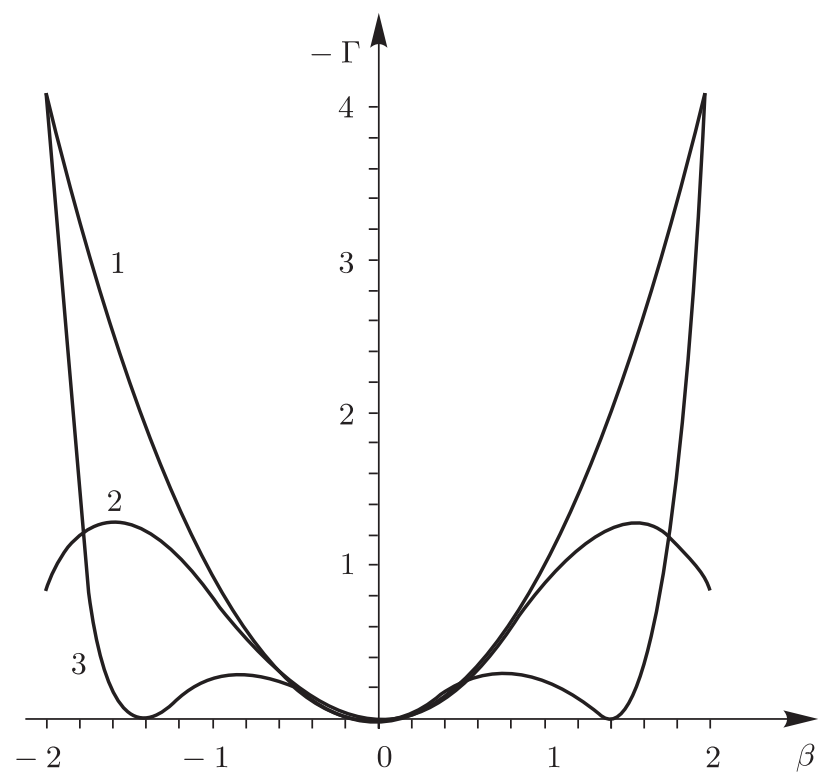

Рис. 2. Вид термодинамического потенциала как функции параметра порядка.

старших петель дают лишь $\epsilon$-поправки к результатам теории среднего поля. Но теперь заряды в уравнении (13) должны быть заменены на инвариантные $\bar{g}_{i}$, которые, в свою очередь, зависят от параметра $\tau$. При уменьшении $\tau$ инвариантные заряды изменяются, как показано на рис. 1, и при некотором $\tau_{0}>0$ пересекают границу области устойчивости. При этом появляется новое решение уравнения стационарности (13). Отметим, что все это происходит при температуре, большей $T_{\mathrm{c}}$, предсказанной уравнением Дайсона (2), так что описываемое явление в некотором смысле можно назвать высокотемпературной сверхпроводимостью (напомним, что появление аномального решения уравнения стационарности приводит к сверхпроводимости в системе [1]).

Конечно, нельзя утверждать, что $\tau_{0}$ определяет температуру фазового перехода первого рода; при $\tau=\tau_{0}$ в системе лишь появляются метастабильные состояния. Это полезно проиллюстрировать графиком термодинамического потенциала, соответствующего преобразованию Лежандра -Г (см. рис. 2). Термодинамический потенциал $-\Gamma$ имеет вид, изображенный кривой 1 при $\tau>\tau_{0}$; при $\tau=\tau_{0}$ появляется максимум (см. кривую 2). Точке фазового перехода первого рода соответствует линия 3.

Для ответа на вопрос, когда новое состояние системы (с конденсатом) действительно становится стабильным, т. е. для определения температуры фазового перехода, в рассмотрение требуется включить старшие члены действия (9) (типа $\chi^{6}, \chi^{8}, \ldots$ ) и провести РГ-анализ, учитывая их, например, в качестве составных операторов. Такие исследования мы надеемся провести в будущем.

Благодарности. Настоящая работа выполнена при поддержке РФФИ (грант № 12-02-00874_а) и Academy of Finland 2012 (грант № 260934). 


\section{Список литературы}

[1] А. А. Абрикосов, Л. П. Горьков, И. Е. Дзялошинский, Методы квантовой теории поля в статистической физике, Добросвет, М., 2006.

[2] A. I. Larkin, A. A. Varlamov, "Fluctuation phenomena in superconductors", Handbook on Superconductivity: Conventional and Unconventional Superconductors, eds. K. H. Bennemann, J. B. Ketterson, Springer, Berlin, 2002, 369-458, arXiv: cond-mat/0109177.

[3] А.Н. Васильев, Квантовополевая ренормгруппа в теории критического поведения и стохастической динамике, Изд-во ПИЯФ, СПб., 1998.

[4] А.Н. Васильев, Функииональные методы в квантовой теории поля и статистике, ЛГУ, Л., 1976. 